\title{
Wormholes in higher dimensional space-time: Exact solutions and their linear stability analysis
}

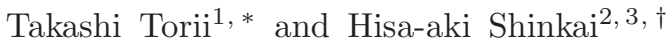 \\ ${ }^{1}$ Department of General Education, Osaka Institute of Technology, Asahi-ku, Osaka 535-8585, Japan \\ ${ }^{2}$ Department of Information Systems, Osaka Institute of Technology, Kitayama, Hirakata, Osaka 573-0196, Japan \\ ${ }^{3}$ Computational Astrophysics Laboratory, Institute of Physical 8 Chemical Research (RIKEN), \\ Hirosawa, Wako, Saitama, 351-0198 Japan
}

(Dated: August 28, 2018)

\begin{abstract}
We derive the simplest traversable wormhole solutions in $n$-dimensional general relativity, assuming static and spherically symmetric spacetime with a ghost scalar field. This is the generalization of the Ellis solution (or the so-called Morris-Thorne's traversable wormhole) into a higher-dimension. We also study their stability using linear perturbation analysis. We obtain the master equation for the perturbed gauge-invariant variable and search their eigenvalues. Our analysis shows that all higher-dimensional wormholes have an unstable mode against the perturbations with which the throat radius is changed. The instability is consistent with the earlier numerical analysis in fourdimensional solution.
\end{abstract}

PACS numbers: 04.20.-q, 04.40.-b, 04.50.-h

\section{INTRODUCTION}

Wormholes are popular tools in science fiction as a way for rapid interstellar travel, time machines and warp drives. However, wormholes are also a scientific topic, just after the birth of general relativity.

Historically, a "tunnel structure" in the Schwarzschild solution was first pointed out by Flamm in 1916[1]. Einstein and Rosen 2] proposed a "bridge structure" between black holes in order to obtain a regular solution without a singularity. The name "wormhole" was coined by John A. Wheeler in 1957, and its fantastic applications are popularized after the influential study of traversable wormholes by Morris and Thorne [3].

Morris and Thorne considered "traversable conditions" for human travel through wormholes responding to Carl Sagan's idea for his novel (Contact), and concluded that such a wormhole solution is available if we allow "exotic matter" (negative-energy matter).

The introduction of exotic matter sounds to be unusual for the first time, but such matter appears in quantum field theory and in alternative gravitational theories such as scalar-tensor theories. The Morris-Thorne solution is constructed with a massless Klein-Gordon field whose gravitational coupling takes the opposite sign to normal, which appears in Ellis's earlier work [4], who called it a drainhole, and also in more general framework of scalartensor theories by Bronnikov in the same year [5]. (See a review e.g. by Visser [6] for earlier works; See also e.g. Lobo 7] for recent works).

Since the difference of light bending behavior between the Ellis wormhole and Schwarzschild black hole were reported by Abe [8], the microlensing images with worm-

\footnotetext{
*Electronic address: torii@ge.oit.ac.jp
}

${ }^{\dagger}$ Electronic address: shinkai@is.oit.ac.jp holes are also getting attention from the observational point of view [9, 10].

One of our main motivations in this paper is the dynamical features of wormholes. A wormhole is supposed to connect two spacetimes as a two-way interface, while a black hole is an one-way interface. From this analogy, Hayward 11] proposed a unified understanding of black holes and traversable wormholes, i.e. a wormhole throat can be interpreted as a degenerate horizon. This idea predicts that a wormhole changes to a black hole in its dynamical evolutions in the classical process.

This is numerically shown by one of the authors [12]. Using a dual-null formulation for space-time integration, they observed that the wormhole is unstable against Gaussian pulses in either an exotic or normal massless Klein-Gordon field. The wormhole throat suffers a bifurcation of the horizon and either explodes to form an inflationary universe or collapses to a black hole, whether the total input energy is negative or positive, respectively.

These basic behaviors were repeatedly confirmed by other groups [13, 14], together with linear perturbation analysis [15] ${ }^{1}$. The wormhole solutions with a conformal scalar field were reported [5, 17], and their instabilities are shown also using linear perturbation analysis [18]. There are also discussions on the wormhole solutions in alternative/modified gravity (e.g. [19, 20]). Wormhole thermodynamics is also proposed based on these properties [21].

We, therefore, understand that four-dimensional Ellis wormhole is unstable. If this feature can also be seen in higher-dimensional spacetime, it should be generic inde-

1 Armendariz-Picon [16] reported that the Ellis wormhole is stable using perturbation analysis. However, Gonzalez et al 15] reported that his conclusion is within the limited class of perturbations and the Ellis wormhole is unstable 
pendent of the dimension. The higher-dimensional theories such as string/M theories are applied for various unsolved problems in gravitational phenomena and cosmology, and we gain new insights into them. The wormholes in higher dimensional general relativity lead to the study in such fundamental theories.

Wormhole study in higher-dimensional spacetime is not a new topic. We can find the articles from 1980s [22, 23], and the recent studies include higher-curvature terms (see e.g. [24] and [25] and references therein). Most of the research concerns the solutions and their energy conditions mainly, but to our knowledge there is no general discussion on the stability analysis of the solutions.

In this article, we construct Ellis solutions in higherdimensional general relativity, and study their stability using the linear perturbation technique. The full numerical studies will be shown in our follow up paper.

This paper is organized as follows. In Section II] we derive our higher-dimensional wormhole solutions. In Section III. we show the linear perturbation analysis. The conclusion and discussion are shown in Section IV]

\section{WORMHOLE SOLUTIONS}

We start from the $n$-dimensional Einstein-KleinGordon system

$$
S=\int d^{n} x \sqrt{-g}\left[\frac{1}{2 \kappa_{n}^{2}} R-\frac{1}{2} \epsilon(\nabla \phi)^{2}-V(\phi)\right],
$$

where $\kappa_{n}^{2}$ is a $n$-dimensional gravitational constant. The scalar field $\phi$ can be called as normal (or ghost) field if $\epsilon=1(-1)$.

This action derives the Einstein equation

$$
G_{\mu \nu}=\kappa_{n}^{2} T_{\mu \nu}
$$

where

$$
T_{\mu \nu}=\epsilon\left(\partial_{\mu} \phi\right)\left(\partial_{\nu} \phi\right)-g_{\mu \nu}\left[\frac{1}{2} \epsilon(\nabla \phi)^{2}+V(\phi)\right]
$$

and the Klein-Gordon equation

$$
\square \phi=-\epsilon \frac{d V}{d \phi} .
$$

We consider the space-time with the metric

$$
\begin{aligned}
d s^{2}= & -f(t, r) e^{-2 \delta(t, r)} d t^{2}+f(t, r)^{-1} d r^{2} \\
& +R(t, r)^{2} h_{i j} d x^{i} d x^{j}
\end{aligned}
$$

where $h_{i j} d x^{i} d x^{j}$ represents the line element of a unit $(n-$ 2)-dimensional constant curvature space with curvature $k= \pm 1,0$ and volume $\Sigma_{k}$.

In order to construct a static wormhole solution, we restrict the metric function as $f=f(r), R=R(r), \phi=$ $\phi(r)$, and $\delta=0$. The $(t, t),(r, r)$, and $(t, r)$ components of the Einstein equations, then, become

$$
\begin{aligned}
& -\frac{n-2}{2} f^{2}\left[\frac{2 R^{\prime \prime}}{R}+\frac{f^{\prime} R^{\prime}}{f R}+\frac{(n-3) R^{\prime 2}}{R^{2}}\right]+\frac{(n-2)(n-3) k f}{2 R^{2}}=\kappa_{n}^{2} f\left[\frac{1}{2} \epsilon f \phi^{\prime 2}+V(\phi)\right], \\
& \frac{n-2}{2} \frac{R^{\prime}}{R}\left[\frac{f^{\prime}}{f}+\frac{(n-3) R^{\prime}}{R}\right]-\frac{(n-2)(n-3) k}{2 f R^{2}}=\frac{\kappa_{n}^{2}}{f}\left[\frac{1}{2} \epsilon f \phi^{\prime 2}-V(\phi)\right], \\
& \frac{f^{\prime \prime}}{2}+(n-3) f\left(\frac{R^{\prime \prime}}{R}+\frac{f^{\prime} R^{\prime}}{f R}+\frac{n-4}{2} \frac{R^{\prime 2}}{R^{2}}\right)-\frac{(n-3)(n-4) k}{2 R^{2}}=\kappa_{n}^{2}\left[\frac{1}{2} \epsilon f \phi^{\prime 2}+V(\phi)\right],
\end{aligned}
$$

respectively, and the Klein-Gordon equation becomes

$$
\frac{1}{R^{n-2}}\left(R^{n-2} f \phi^{\prime}\right)^{\prime}=-\epsilon \frac{d V}{d \phi} .
$$

Hereafter, we assume that the scalar field is ghost $(\epsilon=-1)$ and massless $(V(\phi)=0)$. The Klein-Gordon equation (2.9) is integrated as

$$
\phi^{\prime}=\frac{C}{f R^{n-2}},
$$

where $C$ is an integration constant. The Einstein equa- tions (2.6)-(2.8) are reduced to

$$
\begin{array}{r}
\frac{(n-2) R^{\prime}}{R}\left[\frac{f^{\prime}}{f}+\frac{(n-3) R^{\prime}}{R}\right]-\frac{(n-2)(n-3) k}{f R^{2}} \\
=-\frac{\kappa_{n}^{2} C^{2}}{f^{2} R^{2(n-2)}}
\end{array}
$$

and

$$
\frac{(n-2) R^{\prime \prime}}{R}=\frac{\kappa_{n}^{2} C^{2}}{f^{2} R^{2(n-2)}} .
$$

We assume the throat of the wormhole is at $r=0$, and $a$ is the radius of the throat, i.e., $R(0)=a$. By the 
(a)

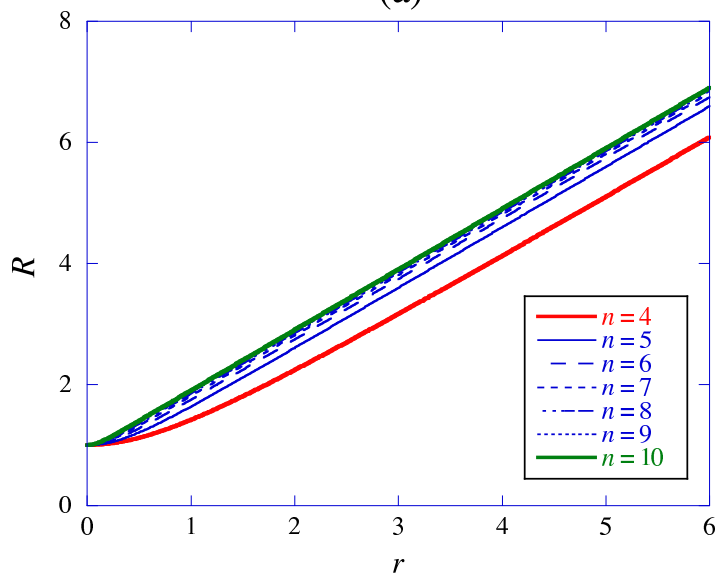

(b)

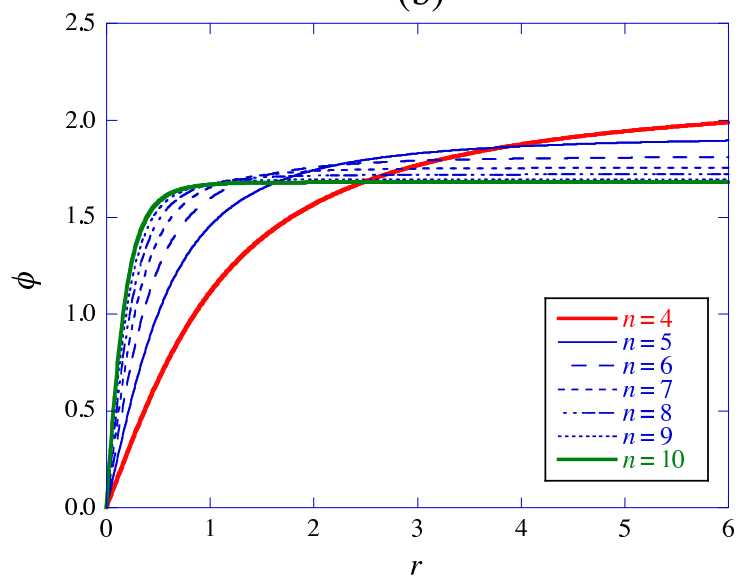

FIG. 1: The $n$-dimensional wormhole solutions; (a) The circumference radius $R$ and (b) the scalar field $\phi$ are plotted as a function of the radial coordinate $r$. The cases of $n=4-10$ are shown.

regularity conditions at the throat,

$$
R(0)=a>0, \text { and } f(0)=f_{0}>0,
$$

where $f_{0}$ is a constant. Here we can assume $a=1$ and $f_{0}=1$ without loss of generality [26], but we keep $a$ in the equations in this section for later convenience. We also assume the reflection symmetry with respect to the throat:

$$
R^{\prime}(0)=0, \text { and } f^{\prime}(0)=0
$$

There is a shift symmetry of the scalar field $\phi$ and we impose $\phi(0)=0$. By substituting these conditions into Eq. (2.11), the integration constant $C$ is determined as

$$
\kappa_{n}^{2} C^{2}=(n-2)(n-3) k a^{2(n-3)} .
$$

For the case $k=0$, the constant $C$ vanishes and the solution becomes trivial. For the case $k=-1$, Eq. (2.15) is not satisfied and there is no wormhole solution. Below we assume $k=1$.

The solution of Eqs. (2.10)-(2.12) is obtained as

$$
\begin{aligned}
f & \equiv 1, \\
R^{\prime} & =\sqrt{1-\left(\frac{a}{R}\right)^{2(n-3)}}, \\
\phi & =\frac{\sqrt{(n-2)(n-3)}}{\kappa_{n}} a^{n-3} \int \frac{1}{R(r)^{n-2}} d r .
\end{aligned}
$$

The Eq. (2.17) is integrated to give

$$
r(R)=-m B_{z}\left(-m, \frac{1}{2}\right)-\frac{\sqrt{\pi} \Gamma[1-m]}{\Gamma[m(n-4)]},
$$

where $m=1 / 2(n-3)$ and $z=R^{m} . \quad B_{z}(p, q)$ is the incomplete beta function defined by

$$
B_{z}(p, q):=\int_{0}^{z} t^{p-1}(1-t)^{q-1} d t
$$

which can be expressed by the hypergeometric function $F(\alpha, \beta, \gamma ; z)$ as

$$
B_{z}(p, q)=\frac{z^{p}}{p} F(p, 1-q, p+1 ; z) .
$$

Although Eq. (2.19) is implicit with respect to $R$, it is rewritten in the explicit form by using the inverse incomplete beta function. For $n=4$, this solution reduces to Ellis's wormhole solution.

$$
f \equiv 1, \quad R=\sqrt{r^{2}+a^{2}}, \quad \phi=\sqrt{2} \tan ^{-1} \frac{r}{a} .
$$

At the throat, we find

$$
R^{\prime \prime}(a)=\frac{n-3}{a}, \text { and } \phi^{\prime}(a)=\frac{\sqrt{(n-2)(n-3)}}{\kappa_{n} a}
$$

These indicate that the throat of the wormhole has larger curvature and the scalar field $\phi$ becomes steeper as $n$ goes higher. At the spacial infinity, the scalar field $\phi(r)$ becomes constant and the function $R(r)$ is proportional to $r$. We plotted these behaviors in Figure 1] For $n \rightarrow \infty$, the functions have the limiting solution, $R=r+a$ and $\phi=\pi / 2(r>0)$. 


\section{STABILITY ANALYSIS}

In this section, we investigate the linear stability of the higher-dimensional wormhole solution obtained in the previous section. In the non-linear analysis in fourdimensional spacetime, it is shown that the instability occurs by resolution of the degeneracy of a double trapping horizon by perturbing the throat radius [12]. Hence we follow the analysis in Ref. [15], where the throat radius is not fixed.

We focus on the "spherical" modes, where the $(n-2)$ dimensional constant curvature space is not perturbed [27]. In the time-dependent and spherically symmetric spacetime, the metric is written as Eq. (2.5) generally. We write the perturbed functions as

$$
\begin{aligned}
f(t, r) & =f_{0}(r)+\varepsilon f_{1}(r) e^{i \omega t}, \\
\delta(t, r) & =\delta_{0}(r)+\varepsilon \delta_{1}(r) e^{i \omega t}, \\
R(t, r) & =R_{0}(r)+\varepsilon R_{1}(r) e^{i \omega t}, \\
\phi(t, r) & =\phi_{0}(r)+\varepsilon \phi_{1}(r) e^{i \omega t} .
\end{aligned}
$$

$\varepsilon$ is an infinitesimal parameter. The variables with subscript 0 denote the static solution obtained in the previous section. This ansatz contains one gauge mode.

The first-order equations of the Einstein equations become

$$
\begin{aligned}
& R_{1}^{\prime \prime}+\frac{(n-3) R_{0}^{\prime}}{R_{0}} R_{1}^{\prime}+\frac{R_{0}^{\prime}}{2} f_{1}^{\prime}+\frac{(n-3)}{2 R_{0}} f_{1}-\sqrt{\frac{n-3}{n-2}} \frac{1}{R_{0}^{n-3}} \phi_{1}^{\prime}=0, \\
& \frac{(n-3) R_{0}^{\prime}}{R_{0}} R_{1}^{\prime}+\frac{n-3}{R_{0}^{2 n-4}} R_{1}+\frac{R_{0}^{\prime}}{2} f_{1}^{\prime}+\frac{(n-3)}{2 R_{0}} f_{1}-R_{0}^{\prime} \delta_{1}^{\prime}+\sqrt{\frac{n-3}{n-2}} \frac{1}{R_{0}^{n-3}} \phi_{1}^{\prime}+R_{1} \omega^{2}=0, \\
& 2 R_{1}^{\prime}-R_{0}^{\prime} f_{1}-2 \sqrt{\frac{n-3}{n-2}} \frac{1}{R_{0}^{n-3}} \phi_{1}=0,
\end{aligned}
$$

for the $(t, t),(r, r)$, and $(t, r)$ components, respectively. Here we assume $a=1$. From Eq. (3.7), $f_{1}$ is

$$
f_{1}=2 \sqrt{\frac{n-3}{n-2}} \frac{1}{R_{0}^{n-3} R_{0}^{\prime}} \phi_{1}-\frac{2}{R_{0}^{\prime}} R_{1}^{\prime} .
$$

By substituting Eq. (3.8) into Eqs. (3.5) and (3.6), we find

$$
R_{1}^{\prime \prime}-\frac{n-3}{R_{0}^{2 n-4}} R_{1}+R_{0}^{\prime} \delta_{1}^{\prime}-2 \sqrt{\frac{n-3}{n-2}} \frac{1}{R_{0}^{n-3}} \phi_{1}^{\prime}=\omega^{2} R_{1} .
$$

With Eq. (3.8), the Klein-Gordon equation turns out to be

$$
\begin{aligned}
\phi_{1}^{\prime \prime}+ & \frac{(n-2)+(n-4) R_{0}^{-2 n+6}}{R_{0} R_{0}^{\prime}} \phi_{1}^{\prime}-\frac{2(n-3)^{2}}{R_{0}^{2 n-4} R_{0}^{\prime 2}} \phi_{1}-\frac{2 \sqrt{(n-2)_{3}}}{R_{0}^{n-2} R_{0}^{\prime}} R_{1}^{\prime \prime} \\
& +\frac{\sqrt{(n-2)_{3}}\left[(n-2)+(n-4) R_{0}^{-2 n+6}\right]}{R_{0}^{n-1} R_{0}^{\prime 2}} R_{1}^{\prime}-\frac{(n-2) \sqrt{(n-2)_{3}} R_{0}^{\prime}}{R_{0}^{n}} R_{1}-\frac{\sqrt{(n-2)_{3}}}{R_{0}^{n-2}} \delta_{1}^{\prime}=-\omega^{2} \phi_{1} .
\end{aligned}
$$

By introducing the new variable,

$$
\psi_{1}=R_{0}^{\frac{n-2}{2}}\left(\phi_{1}-\frac{\phi_{0}^{\prime}}{R_{0}^{\prime}} R_{1}\right),
$$

we find Eqs. (3.9) and (3.10) give the single master equation,

$$
-\psi_{1}^{\prime \prime}+V(r) \psi_{1}=\omega^{2} \psi_{1}
$$

with the potential,

$$
V(r)=\frac{n-2}{2}\left[\frac{n-3}{R_{0}^{2(n-2)}}+\frac{(n-4) R_{0}^{\prime 2}}{2 R_{0}^{2}}\right]+\frac{2(n-3)^{2}}{R_{0}^{2(n-2)} R_{0}^{\prime 2}} .
$$

The variable $\psi_{1}$ is gauge invariant under the spherically symmetric ansatz. However, $R_{0}^{\prime}$ is zero at the throat, and the potential $V$ diverges there. Hence we regularize the master equation (3.12) 28].

It is easily checked that the master equation (3.12) has a 0 -mode solution

$$
\bar{\psi}_{1}=\frac{1}{R_{0}^{\frac{n-4}{2}} R_{0}^{\prime}} .
$$

With the 0-mode solution, we define differential operators

$$
\mathcal{D}_{+}=\frac{d}{d r}-\frac{\bar{\psi}_{1}^{\prime}}{\bar{\psi}_{1}} \quad \text { and } \quad \mathcal{D}_{-}=-\frac{d}{d r}-\frac{\bar{\psi}_{1}^{\prime}}{\bar{\psi}_{1}}
$$




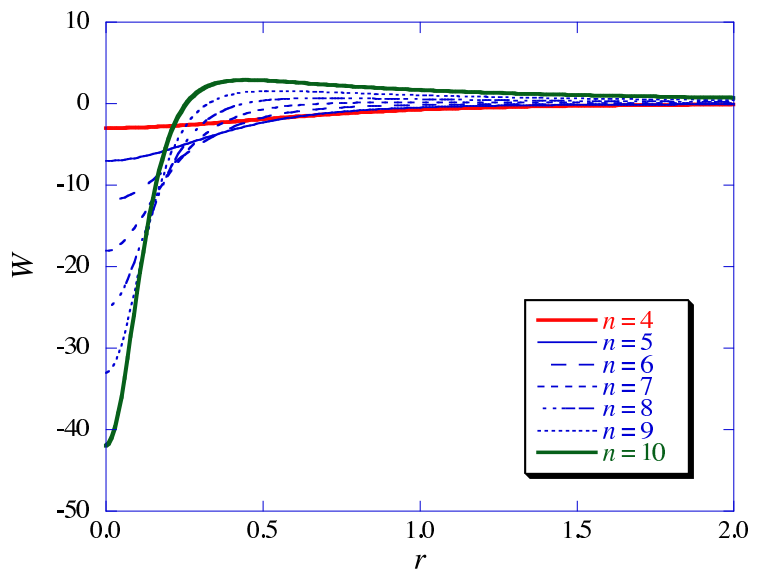

FIG. 2: The potential function $W(r)$ is plotted. $W(r)$ is finite everywhere, and negative around the throat.

Then the master equation, (3.12), can be written as

$$
\mathcal{D}_{-} \mathcal{D}_{+} \psi_{1}=\omega^{2} \phi_{1} \text {. }
$$

Operating $\mathcal{D}_{+}$from the left and defining the new variable $\Psi_{1}=\mathcal{D}_{+} \psi_{1}$, we find the regularized master equation

$$
-\Psi_{1}^{\prime \prime}+W(r) \Psi_{1}=\omega^{2} \Psi_{1}
$$

where

$$
W(r)=-\frac{1}{4 R_{0}^{2}}\left[\frac{3(n-2)^{2}}{R_{0}^{2(n-3)}}-(n-4)(n-6)\right] .
$$

Figure 2 shows the configurations of the potential function $W(r)$. Now the potential function is regular everywhere. For $n=4, W(r)$ has the minimum at the throat and is negative definite. For $n \geq 5, W(r)$ has the minimum at the throat, while it increases apart from the throat and becomes positive for large $r$.

We search the eigenfunctions $\Psi_{1}(r)$ of Eq. (3.17), and find them in any dimension $n$. There exists one negative eigenvalue for $\omega^{2}$, which are listed in Table \. The existence of the eigenfunction with negative $\omega^{2}$ implies that the solution is unstable. We find large negative $\omega^{2}$ for higher $n$, which indicates the time-scale of instability becomes shorter. This feature corresponds to the depth of the potential $W$. The associated eigenfunctions $\Psi_{1}(r)$ are shown in Figure 3 .

\section{CONCLUSIONS AND DISCUSSIONS}

We derived the simplest wormhole solutions in higherdimensional general relativity. The spacetime is assumed to be static and spherically symmetric, has ghost scalar field, and has reflection symmetry at the throat. The four-dimensional version is known as the Ellis (MorrisThorne) solution. At the throat, both the ingoing and outgoing expansions vanish, which means that the throat consists of a degenerate horizon.

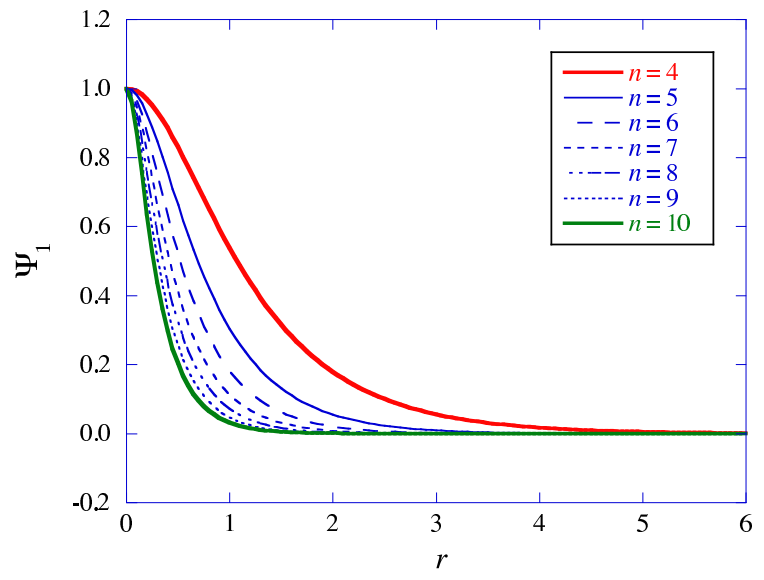

FIG. 3: The eigenfunction $\Psi_{1}$ [Eq. 3.17] $]$ is plotted as a function of radial coordinate $r$.

TABLE I: The negative eigenvalues $\omega^{2}$.

\begin{tabular}{rc}
\hline$n$ & $\omega^{2}$ \\
\hline 4 & -1.39705243371511 \\
5 & -2.98495893027790 \\
6 & -4.68662054299460 \\
7 & -6.46258414126318 \\
8 & -8.28975936306259 \\
9 & -10.1535530451867 \\
10 & -12.0442650147438 \\
11 & -13.9552091676647 \\
20 & -31.5751101285105 \\
50 & -91.3457759137153 \\
100 & -191.283017729717 \\
\hline
\end{tabular}

The obtained solutions are expressed with the incomplete beta function. We expect that the solution can be expressed more simple functional form if we use another coordinate system. Or such an expression might have appeared in the literature, but we have not noticed it. However, we believe the successive stability analysis is new to us.

From the stability analysis using the linear perturbation technique, we showed that the solutions have one negative mode, which concludes that all wormholes are linearly unstable. The time scale of instability becomes shorter as $n$ becomes large.

By extrapolating the knowledge of four-dimensional Ellis's wormhole, we expect that these higherdimensional wormholes also change to a black hole or an expanding throat. This is actually true. In our succeeding papers, we will report the numerical evolutions of higher-dimensional wormholes, in which we show the above predictions are realized. Both linearly perturbed solutions and solutions with nonlinear pulse input suffer the bifurcations of horizons and turn to either black hole or expanding throat. In order to obtain a robust wormhole solution for such a disturbance, we may have to work in modified gravity theories, as was recently reported in dilaton-Gauss-Bonnet gravity [25]. 
The instability of wormholes requires additional maintenance techniques in science fiction. Not only so, but this indicates that such a simple wormhole construction cannot be available as an astrophysical object with the present setting.

\section{Acknowledgements}

T.T. thanks Ken Kamano, Hideki Maeda, Umpei Miyamoto, Masaaki Morita and Makoto Narita for use- ful discussions. We also thank the anonymous referee for pointing out earlier references. This work was supported in part by the Grant-in-Aid for Scientific Research Fund of the JSPS (C) No. 22540293, and (C) No. 25400277. Numerical computations were carried out on SR16000 at YITP in Kyoto University, and on the RIKEN Integrated Cluster of Clusters (RICC).
[1] L. Flamm, Physik Z. 17, 448 (1916).

[2] A. Einstein and N. Rosen, Phys. Rev. 48, 73 (1935).

[3] M. S. Morris \& K. S. Thorne, Am. J. Phys. 56, 395 (1988).

[4] H. G. Ellis, J. Math. Phys. 14, 395 (1973).

[5] K. A. Bronnikov. Acta Phys. Polon. B4, 251 (1973).

[6] M. Visser, Lorentzian Wormholes (AIP Press, 1995).

[7] F. S. N. Lobo, in Classical and Quantum Gravity Research Progress (Nova Sci. Pub., 2008). arXiv:0710.4474

[8] F. Abe, Astrophys. J. 725, 787 (2010) .

[9] Y. Toki, T. Kitamura, H. Asada, \& F. Abe, Astrophys. J. 740, 121 (2011) .

[10] C-M. Yoo, T. Harada, \& N. Tsukamoto. Phys. Rev. D 87, 084045 (2013).

[11] S A Hayward, Int. J. Mod. Phys. D 8, 373 (1999).

[12] H. Shinkai \& S.A. Hayward, Phys. Rev. D 66, 044005 (2002).

[13] A. Doroshkevich, J. Hansen, I. Novikov, A. Shatskiy, Int. J. Mod. Phys. D 18, 1665 (2009).

[14] J. A. Gonzalez, F. S. Guzman \& O. Sarbach, Class. Quant. Grav. 26, 015011 (2009).

[15] J. A. Gonzalez, F. S. Guzman \& O. Sarbach, Class. Quant. Grav. 26, 015010 (2009).

[16] C. Armendariz-Picon, Phys. Rev. D 65, 104010 (2002).
[17] C. Barceló and M. Visser, Class. Quant. Grav. 17, 3843 (2000).

[18] K.A. Bronnikov, S.V. Grinyok, Grav. Cosmol. 10, 237 (2004). gr-qc/0411063.

[19] K.A. Bronnikov, R.A. Konoplya, A. Zhidenko, Phys. Rev. D 86, 024028 (2012).

[20] I. Novikov, A. Shatskiy, JETP 141, 801 (2012) arXiv:1201.4112

[21] S. A. Hayward, Phys. Rev. D 79, 124001 (2009).

[22] A. Chodos and S. Detweiler, Gen. Rel. Grav. 14, 879 (1982).

[23] G. Clément, Gen. Rel. Grav. 16, 131 (1984).

[24] H. Maeda \& M. Nozawa, Phys. Rev. D 78, 024005 (2008).

[25] P. Kanti, B. Kleihaus \& J. Kunz, Phys. Rev. Lett. 107, 271101 (2011); Phys. Rev. D 85, 044007 (2012).

[26] Introducing the new variables $\tilde{t}=t / a, \tilde{r}=r / a, \tilde{R}=R / a$, we can scale out the throat radius $a$.

[27] In the higher-dimensional spacetime, the constant curvature spaces with $k=1$ are not only the spherically symmetric but there are other spaces such as the one with Bohm metric. However, here we will call the modes "spherical".

[28] See discussions in Ref. [15] for details. 